\title{
https://helda.helsinki.fi
}

\section{LifeLine dialogues with Roberta}

\section{López, Asier}

Springer

2017

López , A , Ratni , A , Trong , T N , Olaso , J M , Montenegro , S , Lee , M , Haider , F , Schlögl , S , Chollet, G, Jokinen, K, Petrovska-Delacrétaz, D, Sansen, H \& Torres , M I 2017 , LifeLine dialogues with Roberta . in J Quesada ... [et al.] (ed.) , Future and Emerging Trends in Language Technology, Machine Learning and Big Data : Second International pÿWorkshop, FETLT 2016, Seville, Spain, November 30 December 2, 2016, Revised Selected Papers . Lecture Notes in Computer Science (including subseries Lecture Notes in Artificial Intelligence and Lecture Notes in Bioinformatics), vol. 10341, Springer, Cham , pp. 73-85, International Workshop on Future and Emerging Trends in Language Technology , 30/11/2016 . https://doi.org/10.1007/978-3-319-69365-1_6

http://hdl.handle.net/10138/310783

https://doi.org/10.1007/978-3-319-69365-1_6

unspecified

acceptedVersion

Downloaded from Helda, University of Helsinki institutional repository.

This is an electronic reprint of the original article.

This reprint may differ from the original in pagination and typographic detail.

Please cite the original version. 


\title{
LifeLine Dialogues with Roberta
}

\author{
Asier López ${ }^{1}$, Ahmed Ratni ${ }^{2}$, Trung Ngo Trong ${ }^{3}$, Javier Mikel Olaso ${ }^{1}$, \\ Seth Montenegro ${ }^{4}$, Minha Lee ${ }^{5}$, Fasih Haider ${ }^{6}$, Stephan Schlögl ${ }^{7}$, \\ ${\text { Gérard } \text { Chollet }^{8} \text {, Kristiina Jokinen }}^{3}$, Dijana Petrovska-Delacrétaz ${ }^{2}$, \\ Hugues Sansen ${ }^{9}$, and María Inés Torres ${ }^{1(\bowtie)}$ \\ 1 Universidad del País Vasco (UPV/EHU), Bilbao, Spain \\ manes.torres@ehu.eus \\ 2 CNRS SAMOVAR UMR 5157, Télécom SudParis, \\ Université Paris-Saclay, Paris, France \\ 3 University of Helsinki, Helsinki, Finland \\ ${ }^{4}$ Hyx, London, UK \\ ${ }^{5}$ Eindhoven University of Technology, Eindhoven, The Netherlands \\ 6 Trinity College Dublin, University of Dublin, Dublin, Ireland \\ 7 Management Center Innsbruck, Innsbruck, Austria \\ 8 Intelligent Voice, London, UK \\ 9 Shankaa, Paris, France
}

\begin{abstract}
This paper describes work on dialogue data collection and dialogue system design for personal assistant humanoid robots undertaken at eNTERFACE 2016. The emphasis has been on the system's speech capabilities and dialogue modeling of what we call LifeLine Dialogues, i.e. dialogues that help people tell stories about their lives. The main goal behind this type of application is to help elderly people exercise their speech and memory capabilities. The system further aims at acquiring a good level of knowledge about the person's interests and thus is expected to feature open-domain conversations, presenting useful and interesting information to the user. The novel contributions of this work are: (1) a flexible spoken dialogue system that extends the Ravenclawtype agent-based dialogue management model with topic management and multi-modal capabilities, especially with face recognition technologies, (2) a collection of WOZ-data related to initial encounters and presentation of information to the user, and (3) the establishment of a closer conversational relationship with the user by utilizing additional data (e.g. context, dialogue history, emotions, user goals, etc.).
\end{abstract}

Keywords: Dialogue system - Speech input - Agent-based dialogue management $\cdot$ Topic trees

\section{Introduction}

Human-robot interaction provides a new and promising area for the study of a dialogue partner's engagement in conversational interactions, and for experimenting with various presentation strategies, their cultural differences and the 
impact on the user's perception, understanding, and evaluation of the interaction. Robots have also opened up novel possibilities in practical applications, e.g. in scenarios which focus on providing tools for the aging population, such as maintaining companionship, detecting health problems, and assisting dependent people. In addition, adaptation to the user can be learned. Roberta Ironside is a personal assistant humanoid robot project that emphasizes speech capabilities for interacting with dependent persons. The project covers: speech processing and dialogue management, affective computing, human behavior analysis, and human robot interaction and assistance. In this paper we focus on the development of Roberta's LifeLine Dialogues, which was one of the topics pursued during the eNTERFACE 2016 workshop series held at the University of Twente, The Netherlands ${ }^{1}$. During four weeks, a team consisting of research students and a number of faculty members worked on different dialogues for Roberta. The results of this undertaking are summarized below. A more extended version of this paper will be available later this year, or early next year, as a report published in the digital CTIT proceedings of the University of Twente.

As a start we worked on dialogue management. The task was to produce clear, natural, engaging, and intuitively easy-to-follow mixed-initiative interactions where both the user and the system are engaged in satisfying and smooth conversational-type situations. While the long-term goal of Roberta Ironside is that both the human as well as the robot can initiate topics and ask questions, for this workshop we focused on the initial encounter and presentation dialogues. In addition to spoken input Roberta was able to obtain information from the environment (incl. an interlocutor's face) and from the system through a dialogue manager that integrates this information within its dialogue tasks and strategies. It was designed to help people tell stories about their lives and consequently help them exercise their speech and memory capabilities. It further aims at acquiring a good level of knowledge about the person and his/her context and thus is expected to feature an open-domain conversational system, presenting useful and interesting information to the user. The novel contributions of this project can thus be summarized in providing a real world application:

- to build a robot-driven spoken dialogue system that integrates and improves face recognition technologies into its dialogue models, and

- to utilize these data (e.g. context, dialogue history, emotions, user goals, etc.) so as to establish a closer conversational relationship with the user, by considering both knowledge of the user as well as the dialogue history.

\section{Background}

A Spoken Dialogue System (SDS) is a collection of natural language processing components which enables speech-based interaction between a user and a situated agent or knowledge base. Multimodal dialogue systems extend the basic notion of a spoken dialogue system with additional input and output modalities

\footnotetext{
${ }^{1}$ http://hmi.ewi.utwente.nl/enterface16/.
} 
such as visual, tactile, or biosensor information, and thus need extra processing components to take care of sensor fusion and sensor fission.

The basic tasks around dialogue management include: (1) interpretation of the user utterance in a given context (i.e. Natural Language Understanding), (2) decision of what action the system should take (i.e. actual Dialogue Management), and (3) production of an appropriate response (Natural Language Generation).

Moreover, the dialogue manager component keeps track of the dialogue context by integrating the recognized user messages as well as the produced system messages into the dialogue history. Often a separate User Model (UM) is provided to record the user specific characteristics and preference information, and a specific World Model (WM) to store the general knowledge of the world and the environment.

An important part of the dialogue manager is the handling of the grounding process, i.e. overseeing the way how information uttered by the participants is used to form 'common ground', or assumed to be mutually known by the participants $[2,20]$. This requires that interlocutors actively contribute to the dialogue and let their partner(s) know that they have heard and accepted their contribution. Feedback may be verbal acknowledgments and/or non-verbal communicative acts like head nods, facial expressions and other gestures.

The dialogue manager decides upon the action a system should perform at any given state in the dialogue. Common dialogue techniques are described in [9] where script-based, frame-based, agent-based, and statistical dialogue management models are distinguished.

Script-based dialogue management designs the dialogue as a state diagram where each state and dialogue action are paired. Although easy to design and implement for simple dialogue tasks, they require significant effort to be built and maintained for complex dialogues. A frame-based DM provides more flexible dialogue management by separating knowledge from dialogue actions, so that the actions can be executed in various orders depending on the current information state (e.g. [19,21]). The frame also provides a dialogue context. However, more extended control algorithms and natural language grammar capabilities are necessary.

Many advanced dialogue management architectures use an agent-based approach like the one employed by CMU's Ravenclaw [1]. Originally developed in the Galaxy Communicator project, and further enhanced by [11], Ravenclaw provides an advanced management structure with distributed software agents; i.e. dialogue flow can be modeled by the software agents' reasoning about their own state and the next action. Dialogue structure and reasoning can be flexibly designed, and it is also possible to take multimodal information and dynamic context into account. Similar architectures have been proposed for example in TRIPS [5], EDECAN [12] and JASPIS [22].

Interactive systems are an ongoing field of research, in particular such applications that face the challenges of real world users $[4,7,18]$ and those that deal with adaptive and multi-modal systems. For the initial work on Roberta Ironside we have focused on building a basic dialogue system based on Ravenclaw and from there study more advanced issues in a systematic way. 


\section{System Architecture}

The system we developed consists of an input perception layer, an understanding module, an interaction module that includes the dialogue manager which also manages a user blackboard, a dialogue history blackboard and an open domain conversational agent that gets information from a digital repository and a final output layer. While eventually all these components will be integrated into the body of a humanoid robot, our work group at eNTERFACE 2016 focused solely on the spoken interaction aspects, for which the physical system representation was currently neglected.

\subsection{Perception and Understanding}

Automatic Speech Recognition - For Automatic Speech Recognition (ASR) our goal was to distribute processing between a local and a remote module. Remote access should be used to allow for richer linguistic data and knowledge processing (note: at eNTERFACE 2016 we used remote servers of Intelligent Voice ${ }^{2}$ and Google ${ }^{3}$ ). Locally, an HMM/DNN decoder running on an NVIDIA GPU platform should serve as a backup. Although a number of different languages were available, at eNTERFACE 2016 we focused on English.

Audio-Visual Signal Processing - As for audio-visual signal processing we used the SudFrog (Telecom SudParis Face Recognition open-source reference software $)^{4}$. It is part of the open-source reference systems initially developed by the BioSecure Network of excellence [13], and has been extensively tested on different large scale and challenging biometric databases, such as the FRGC [14] and the MOBIO [10] databases. Recently it has further been implemented in an audio-visual verification application running on the iPad [23]. It offers $2 \mathrm{D}$ face recognition, speaker verification as well as the synchronization of lip movements with the speech signal. At eNTERFACE 2016 we used SudFrog to add an additional input channel, which should help us contextualize interactions. That is, a number of our study participants enrolled into a dialogue with Roberta with their faces. The enrollment phase consisted of the face detection, the normalization, and the creation of a template that represented the subject for further comparisons. In case of a second encounter with the same participant, Roberta would check whether she would 'recognize' this face, and if yes would provide the dialogue manager with the name of the participant.

Natural Language Understanding - With respect to Natural Language Understanding (NLU), we have seen that a semantic parser that uses statistical classifiers outperforms semantic decoders that use manually constructed semantic grammars [8]. However, such a parser needs to be trained by a sufficient number of sentences representing the task. Thus, a main goal of our eNTERFACE 2016

\footnotetext{
${ }^{2}$ http://www.intelligentvoice.com/.

${ }^{3}$ https://cloud.google.com/speech/.

${ }^{4}$ http://share.int-evry.fr/svnview-eph/.
} 
efforts was to collect a respective dataset of sentences, and transform them in corresponding grammar rules and concepts using the Phoenix Semantic Parser [24]. We were able to collect data from interactions with 15 participants, which gave us some initial constructs and vocabulary to work with. Additional interactions increasing the scope of our dialogues are planned for the future.

\subsection{Interaction and Dialogue Management}

As already mentioned above, our system used Ravenclaw to offer independent agenda-based dialogue management. Ravenclaw is a plan-based and taskindependent framework for dialogue management. Domain specific logic is designed through a task specification level using a tree of dialogue agents. A domain-independent dialogue engine then executes the specified dialogue task and thus controls the dialogue [1]. The dialogue specification task is just a plan for interaction described as a hierarchy of sub-dialogues. A small set of fundamental dialogue agents are located at terminal positions of the tree. This set consists of an Inform, an Expect, a Request and an Execute agent that produces output, expects information, requests information and performs a domain-specific action. Then dialogue-agencies occupy non-terminal positions in the tree, i.e. representing sub-tasks or sub-dialogues. The execution of agencies is controlled by preconditions, triggers and success and failure criteria. An important feature of Ravenclaw is that the information flow trough the tree is encapsulated in concepts. A more detailed description of this can be found in [1]. Using Ravenclaw allowed us to design a flexible topic dependent dialogue manager, extending the initial capabilities of this framework.

\subsection{Output}

The goal was to provide facial animation in sync with text-to-speech output. For this we used Unity's game engine. We started by using GRFL's Base Liza 3D model $^{5}$. This saved time since it included animation clips for facial expressions and pre-generated viseme articulations. The phonemes served by the MaryTTS text-to-speech instance were then mapped to their matching visemes for the English language and synchronized with the 3D animations. From there we just had to integrate it with ActiveMQ (the messaging service we used to connect all our components) and push the messages to be read aloud by the Linux build of Roberta's Unity project.

Figure 1 shows the main components of the Roberta dialogue framework developed at eNTERFACE 2016.

\section{Audiovisual Dialogues Through Wizard of $\mathrm{Oz}$}

In order to design and shape interactions with complex technical solutions such as the ones envisaged by Roberta Ironside, researchers require tools and methods

\footnotetext{
${ }^{5}$ https://www.assetstore.unity3d.com/en/\#!/content/52234.
} 


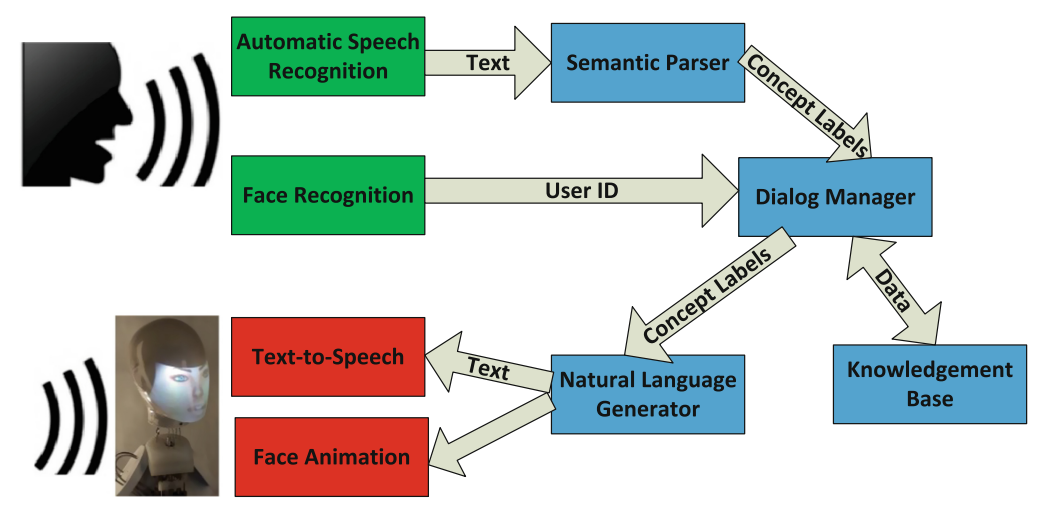

Fig. 1. Main modules of Roberta developed at eNTERFACE 2016

that allow for the early stage prototyping and evaluation of potential development directions. One approach often used for such low-fidelity evaluations is Wizard of $\mathrm{Oz}$ (WOZ) [3]. WOZ constitutes a prototyping method employed by researchers and designers to obtain initial feedback on features that usually require significant resources to be implemented. In a so called 'WOZ experiment' a human 'wizard' mimics the functionality of a system, either entirely or in part, which allows for the evaluation of potential user experiences and interaction strategies without the need for building the actual product. Language based interaction with systems has been one of the main fields of application for this type of prototyping, as building high-end prototypes in this domain tends to be rather costly and time intensive. For example, in order to be able to obtain a basic understanding of an envisioned spoken interaction, systems may require several hours of recorded speech, valid transcriptions, and an implemented framework of rules, as well as a solid error-recovery strategy. Obtaining these types of resources usually requires several person months of work. Here WOZ experimentation can help collect data as well as test overall design directions. The two main ingredients required to successfully conduct WOZ experiments are (1) a prototyping framework/software that allows for the creation of a more or less realistic experimental setting (i.e. the wizard should be hidden from test participants so that they may feel as if they would be interacting with a real system) and (2) a set of scenarios which reflect the capabilities and features of the envisioned future solution. Both was available for our work at eNTERFACE 2016.

\subsection{The WOZ Prototyping Tool}

We used the WebWOZ prototyping platform ${ }^{6}$, a tool specifically geared towards designing and evaluating natural language based interactions [16]. It is entirely web-based and allows for the integration of existing language technologies such as automatic speech recognition and text-to-speech synthesis modules. In order

\footnotetext{
${ }^{6}$ https://github.com/stephanschloegl/WebWOZ.
} 
to collect the above mentioned interaction data (i.e. an initial corpus based on 15 participants) and to obtain a general understanding of potential user strategies we used a pre-configured version of WebWOZ running within a VirtualBox system image [17]. The system used the Google Speech API ${ }^{7}$ for speech recognition to provide the input to the wizard and the Open-Mary TTS engine ${ }^{8}$ for synthesis. The dialogue management at this stage was entirely wizard-based. That is, we prepared a number of utterances from which the wizard could choose so as to engage a participant into a dialogue. Initially those utterance came from how we expected the envisioned dialogue would progress. Yet, each experiment produced a new set of what one may call 'realistic' utterances, which gradually increased our 'interaction pool' and also produced new insight with respect to potential dialogue strategies (note: utterances could also be produced on the fly so that the wizard was not restricted to the initial set). While our long-term goal for Roberta is to offer the possibility for open domain conversations, this initial design stage was focused on a limited number of interaction scenarios.

\subsection{WOZ Driven Roberta Scenarios}

Flandorfer advises on having an interdisciplinary team in constructing user scenarios to better understand users' needs instead of just typecasting potential users [6]. We followed this advice and employed a team with diverse expertise including engineers and human-computer interaction researchers. We first wanted to know what kind of personal data we can gather through the intake conversation with Roberta. We used WebWOZ to conduct a study with 15 volunteers on four pre-identified topics i.e. (1) introduction, (2) professional life, (3) hobbies, and (4) conversation closing. These topics constitute the starting points of building a unified storyline about a person, which is the overall aim of the LifeLine module Roberta should eventually be assembled with.

Dahlbäck et al. [3] described the importance of learning from real-life conversations when building natural language dialogue structures because humanmachine interactions are full of unexpected conversational turns [3]. Also, sociodemographic information is an important part of whether or not assistive robots will be accepted by their users, yet various researchers, system designers, and engineers do not incorporate sociodemographic information [6]. Our WOZ study showed that it is possible to identify a user's sociodemographic background during an initial conversation with Roberta. Roberta begins by checking if a person's face is registered in her database. If it is not, then first, Roberta asks for permission to record the new user into her database. Second, Roberta asks for his/her name and spelling of the name when it is not easily identified. Third, she asks where the user is from and where the user now lives. Fourth, she asks about the user's age, although we learned that age can be a sensitive question. Thus, if no answer is given, Roberta does not immediately repeat the question but rather saves it for later. Fifth, she asks for an opinion about the

\footnotetext{
7 https://cloud.google.com/speech/.

${ }^{8}$ http://mary.dfki.de/.
} 
user's hometown and/or his/her place of residence. Roberta was also able to provide some answers about herself (e.g. "I am Roberta"; "I am infinite"; "I'm from Enschede"), which makes information gathering conversational. Below are two sample conversations from our experiments which demonstrate this:

\section{Conversation 1}

Roberta: Hi I am Roberta. Do you want to register and talk with me?

User: Yes I would like that.

$\mathrm{R}$ : What is your name?

$\mathrm{U}$ : John.

$\mathrm{R}$ : Where are you from?

$\mathrm{U}$ : Israel. And you?

R: I'm from Enschede. Do you know it?

U: Yes I do, but I still prefer Israel.

\section{Conversation 2}

R: Hi I am Roberta. Do you want to register and talk with me?

U: Yes, I am here to talk to you. I am glad to know you.

$\mathrm{R}$ : Good. What is your name?

U: I'm Sungjin from Korea.

$\mathrm{R}$ : Can you spell that for me?

U: S-u-n-g-j-i-n.

R: Great. Where do you actually live?

U: Sorry I don't understand your question.

$\mathrm{R}$ : Where do you live?

$\mathrm{U}$ : Ah you mean where do I now live. I live in Amsterdam.

$\mathrm{R}$ : Do you like it there?

U: It's okay. It's nice, but can be challenging.

\subsection{Moving to LifeLine Dialogues}

The pre-defined conversational topics we used for our WOZ study were inspired by the idea of providing Roberta with what we call a LifeLine module. The goal of LifeLine is to help a person tell the story of his/her life [15]. Such offers an infinite number of subjects to talk about while requiring a rather simplistic framework of questions and answers. Also, it helps a person to work on his/her memory capabilities. With LifeLine, we hope that elderly persons will find a permanent ear for discussing about the past. At the same time, the Roberta system obtains more knowledge about the person. LifeLine could thus be seen as a social network where people's life lines could cross and be linked for a certain period of their life. By sharing information about an event, people will be able to enrich the event, which again could be a subject for later discussion.

We have seen that even with very little information gathered from an initial conversation with a user, we are able to build a rich picture of this person, including relevant sociodemographic information. This initial intake conversation is useful for two reasons. First, it begins a user's LifeLine that is semiautobiographic, with Roberta acting as a ghostwriter who categorizes and places 
stories from the user's life in a timeline as she gets to know the user better. Second, Roberta establishes a user's sociodemographic background, which helps tailor conversational approaches to fit each user.

Our study participants at eNTERFACE 2016 may not have been the exact right target group for LifeLine. They all received at least undergraduate education and were more knowledgeable about technology than the average population. Yet, they provided an initial starting point. Going from here, future studies will be conducted with older, less educated users that are potentially less knowledgeable about technology.

\section{$5 \quad$ System Building}

Figure 2 shows the topic-based dialogue structure we developed based on the conducted WOZ study and its gathered data. An agency was defined for each topic defined during the WOZ scenarios. Then the acquired dialogues guided us through the design of agencies. Note that the use of concepts as triggers allowed us to control the execution of each agency or sub-tree so that a change of topic could appear as often as required, resulting in a very natural and flexible topic-based dialogue structure. As an example, if a participant names a place this can trigger a Roberta interest in said place. She is then able to access a knowledge repository to retrieve information of this place, or, in case the place is already an attribute value in the user blackboard or in the history of the dialogue blackboard, she can ask the user about the place, using a request agent.

The WOZ data served as the foundation for developing the "Authentication" and the "Intro" agencies in Fig. 2. On top of that, Roberta can either ask about a user's professional life or hobbies according to the tree shown in Fig. 2. In case the user first talks about his/her professional life, Roberta asks about the person's current job, tasks related to the job, a typical workday, and his/her educational background. If the user does not currently have a job, Roberta asks about what the user used to do or study.

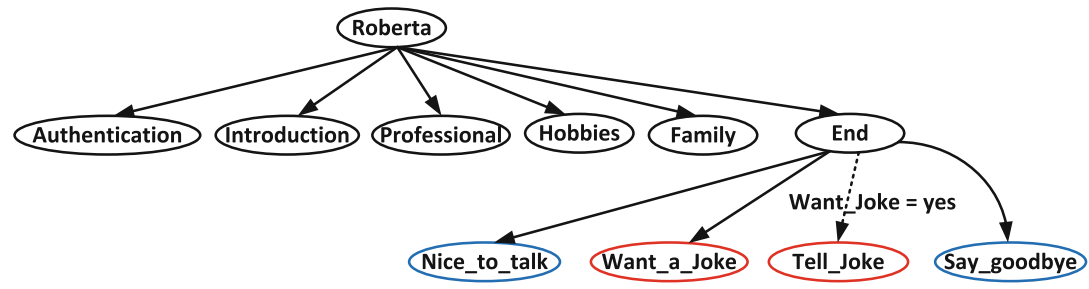

Fig. 2. Topic-based dialogue structure 
Conversation 3

R: What's your job?

U: I'm now working on an airport security project.

R: What tasks do you do?

U: That's very specific. Right now I am working on voice activation.

R: Tell me about your typical workday.

$\mathrm{U}$ : Well I wake up around eight and come to work.

R: Wonderful. What did you study?

U: I studied electrical engineering in France.

Roberta can then ask about hobbies. If the user does not have any hobbies, Roberta will ask more specifically about music, sports or traveling. All three categories have anticipatory questions and/or statements. For example, Roberta will ask "do you like music?", followed by "who is your favorite musician?" or "what is your favorite genre?" or "do you play any instruments?".

Conversation 4

$\mathrm{R}$ : What is your hobby?

$\mathrm{U}$ : I don't have a lot of time for hobbies.

R: Do you like music?

$\mathrm{U}$ : Yes I like music.

$\mathrm{R}$ : What is your favorite genre?

$\mathrm{U}$ : I like classical music.

R: Do you play any instruments?

$\mathrm{U}$ : I used to play piano when I was very very young, but not anymore.

Conversation 5

R: What is your hobby?

$\mathrm{U}$ : I like photography and traveling.

R: Tell me more about the places you've visited.

U: I visited so many countries... Germany, Ireland, England, Mexico, Brazil...

The end of the conversation can also be controlled by some trigger conditions, as shown in Fig. 2. At this point Roberta may tell a joke and/or ask for permission to talk at a later time.

Conversation 6

R: Do you want to listen to a joke?

$\mathrm{U}$ : Yes please.

R: Why was 7 afraid of 9 ?

$\mathrm{U}$ : Why?

R: Because 789 .

$\mathrm{U}$ : Very funny.

R: Do you want to talk more some other time?

$\mathrm{U}$ : Yeah maybe some other time.

R: Talk to you later.

$\mathrm{U}$ : Bye. 


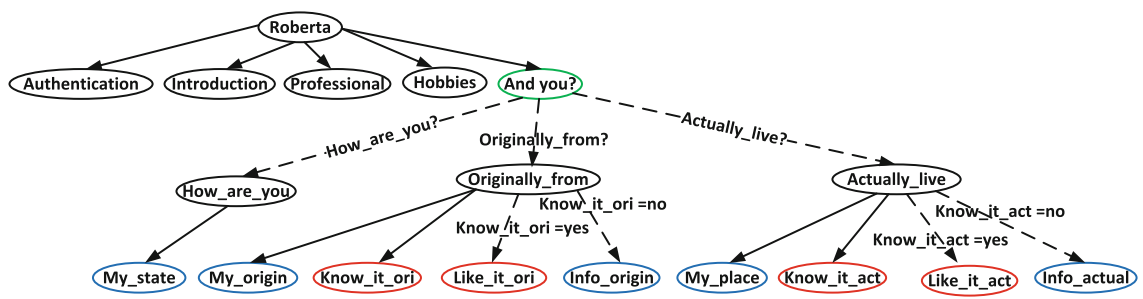

Fig. 3. Allowing for user questions

The WOZ study further showed that some people liked to ask questions back. So we also built an 'and-you' agency. This agency used some expectation agents that added naturalness to the dialogue and some information agents providing Roberta's answers (cf. Fig. 3).

Finally we developed the preliminary design of a feedback agent able to provide Roberta with the capability of giving feedback to the user. It uses inform and execution agents allowing for sufficient dialogue flexibility.

\section{Conversation 7}

R: Where are you originally from?

U: I'm originally from Spain. And you Roberta?

R: Nice question, I'm from the Cybernation.

\section{Concluding Remarks and Future Work}

The paper has described work on dialogue data collection and dialogue system design for personal assistant humanoid robots undertaken at eNTERFACE 2016. The emphasis has been on the system's speech capabilities and dialogue modeling of LifeLine dialogues. Hence, WOZ data based on 15 video recorded conversations is currently available. This collection served as the basis for designing our face recognition, speech understanding, dialogue task and natural language generator modules. The current system includes a flexible spoken dialogue system that extends the initial Ravenclaw dialogue management framework with topic management and multimodal capabilities.

Roberta's LifeLine module is, however, still work-in-progress. Some efforts are currently spent on using the same WOZ study design to compile an audiovisual database in more realistic environments, like in the houses of elderly people, and in several languages with the help of tele-assistance services.

Acknowledgments. The authors want to acknowledge the organizers of eNTERFACE 2016 and the University of Twente for providing the opportunity to develop this project. We also want to acknowledge the institutions supporting some of the authors e.g. the Spanish Science Minister under grant TIN2014-54288-C4, 'ADAPT 13/RC/2106', and the Academy of Finland project Digital Citizens grant number 270082 . 


\section{References}

1. Bohus, D., Rudnicky, A.I.: The RavenClaw dialog management framework: architecture and systems. Comput. Speech Lang. 23(3), 332-361 (2009)

2. Clark, H.H., Schaefer, E.F.: Contributing to discourse. Cogn. Sci. 13(2), 259-294 (1989)

3. Dahlbäck, N., Jönsson, A., Ahrenberg, L.: Wizard of Oz studies: why and how. In: Proceedings of the 1st International Conference on Intelligent User Interfaces, pp. 193-200. ACM (1993)

4. Eskenazi, M., Black, A.W., Raux, A., Langner, B.: Lets Go Lab: a platform for evaluation of spoken dialog systems with real world users. In: InterSpeech (2008)

5. Ferguson, G., Allen, J.F.: TRIPs: an integrated intelligent problem-solving assistant. In: Proceedings of the AAAI/IAAI Conference on Artificial Intelligence/Innovative Applications of Artificial Intelligence, pp. 567-572 (1998)

6. Flandorfer, P.: Population ageing and socially assistive robots for elderly persons: the importance of sociodemographic factors for user acceptance. Int. J. Popul. Res. 2012, Article ID 829835, 13 (2012). doi:10.1155/2012/829835

7. Ghigi, F., Eskenazi, M., Torres, M.I., Lee, S.: Incremental dialog processing in a task-oriented dialog. In: InterSpeech, pp. 308-312 (2014)

8. Henderson, J., Merlo, P., Titov, I., Musillo, G.: Multilingual joint parsing of syntactic and semantic dependencies with a latent variable model. Comput. Linguist. 39(4), 949-998 (2013)

9. Jokinen, K., McTear, M.: Spoken Dialogue Systems, vol. 2. Morgan \& Claypool Publishers, Princeton (2009)

10. McCool, C., Marcel, S., Hadid, A., Pietikäinen, M., Matejka, P., Cernockỳ, J., Poh, N., Kittler, J., Larcher, A., Levy, C., et al.: Bi-modal person recognition on a mobile phone: using mobile phone data. In: 2012 IEEE International Conference on Multimedia and Expo Workshops (ICMEW), pp. 635-640. IEEE (2012)

11. Olaso, J.M., Milhorat, P., Himmelsbach, J., Boudy, J., Chollet, G., Schlögl, S., Torres, M.I.: A Multi-lingual evaluation of the vAssist spoken dialog system. Comparing Disco and RavenClaw. In: International Workshop on Spoken Dialogue Systems (2016)

12. Olaso, J.M., Torres, M.I.: Dialogue system based on EDECÁN architecture. In: Sojka, P., Horák, A., Kopeček, I., Pala, K. (eds.) TSD 2010. LNCS, vol. 6231, pp. 547-551. Springer, Heidelberg (2010). doi:10.1007/978-3-642-15760-8_69

13. Petrovska-Delacrétaz, D., Chollet, G., Dorizzi, B. (eds.): Guide to Biometric Reference Systems and Performance Evaluation. Springer, London (2009). doi:10.1007/ 978-1-84800-292-0

14. Phillips, P.J., Flynn, P.J., Scruggs, T., Bowyer, K.W., Chang, J., Hoffman, K., Marques, J., Min, J., Worek, W.: Overview of the face recognition grand challenge. In: 2005 IEEE Computer Society Conference on Computer Vision and Pattern Recognition (CVPR 2005), vol. 1, pp. 947-954. IEEE (2005)

15. Sansen, H., Torres, M.I., Chollet, G., Glackin, C., Petrovska-Delacretaz, D., Boudy, J., Badii, A., Schlögl, S.: The Roberta IRONSIDE project: a dialog capable humanoid personal assistant in a wheelchair for dependent persons. In: 2016 2nd International Conference on Advanced Technologies for Signal and Image Proceedings (ATSIP), pp. 381-386 (2016)

16. Schlögl, S., Doherty, G., Luz, S.: Wizard of Oz experimentation for language technology applications: challenges and tools. Interact. Comput. 27(6), 592-615 (2015) 
17. Schlögl, S., Milhorat, P., Chollet, G., Boudy, J.: Designing language technology applications: a Wizard of $\mathrm{Oz}$ driven prototyping framework. In: Proceedings of the EACL Conference of the European Chapter of the Association for Computer Linguistics, pp. 85-88 (2014)

18. Serrras, M., Pére, N., Torres, M.I., Del Pozo, A.: Entropy-driven dialog for topic classification: detecting and tackling uncertainty. In: International Workshop on Spoken Dialogue Systems (2016)

19. ter Maat, M., Heylen, D.: Flipper: an information state component for spoken dialogue systems. In: Vilhjálmsson, H.H., Kopp, S., Marsella, S., Thórisson, K.R. (eds.) IVA 2011. LNCS, vol. 6895, pp. 470-472. Springer, Heidelberg (2011). doi:10. 1007/978-3-642-23974-8_67

20. Traum, D.R.: A computational theory of grounding in natural language conversation. Technical report, University of Rochester, Rochester, NY, USA (1994)

21. Traum, D.R., Larsson, S.: The information state approach to dialogue management. In: van Kuppevelt, J., Smith, R.W. (eds.) Current and New Directions in Discourse and Dialogue. Text, Speech and Language Technology, vol. 22, pp. 325353. Springer, Dordrecht (2003)

22. Turunen, M., Hakulinen, J.: Jaspis-a framework for multilingual adaptive speech applications. In: InterSpeech, pp. 719-722 (2000)

23. Usoltsev, A., Petrovska-Delacrétaz, D., Houssemeddine, K.: Full video processing for mobile audio-visual identity verification. In: International Conference on Pattern Recognition Applications and Methods ICPRAM 2016 (2016)

24. Ward, W., et al.: The CMU air travel information service: understanding spontaneous speech. In: Proceedings of the DARPA Speech and Natural Language Workshop, vol. 1, pp. 127-129 (1990) 